\title{
Coherent transient transport of interacting electrons through a quantum waveguide switch
}

\author{
Nzar Rauf Abdullah, ${ }^{1, *}$ Chi-Shung Tang, ${ }^{2}$ Andrei Manolescu, ${ }^{3}$ and Vidar Gudmundsson ${ }^{1, \dagger}$ \\ ${ }^{1}$ Science Institute, University of Iceland, Dunhaga 3, IS-107 Reykjavik, Iceland \\ ${ }^{2}$ Department of Mechanical Engineering, National United University, 1, Lienda, Miaoli 36003, Taiwan \\ ${ }^{3}$ Reykjavik University, School of Science and Engineering, Menntavegur 1, IS-101 Reykjavik, Iceland
}

\begin{abstract}
We investigate coherent electron-switching transport in a double quantum waveguide system in a perpendicular static or vanishing magnetic field. The finite symmetric double waveguide is connected to two semi-infinite leads from both ends. The double waveguide can be defined as two parallel finite quantum wires or waveguides coupled via a window to facilitate coherent electron inter-wire transport. By tuning the length of the coupling window, we observe oscillations in the net charge current and a maximum electron conductance for the energy levels of the two waveguides in resonance. The importance of the mutual Coulomb interaction between the electrons and the influence of two-electron states is clarified by comparing results with and without the interaction. Even though the Coulomb interaction can lift two-electron states out of the group of active transport states the length of the coupling window can be tuned to locate two very distinct transport modes in the system in the late transient regime before the onset of a steady state. A static external magnetic field and quantum-dots formed by side gates (side quantum dots) can be used to enhance the inter-waveguide transport which can serve to implement a quantum logic device. The fact that the device can be operated in the transient regime can be used to enhance its speed.
\end{abstract}

\section{INTRODUCTION}

Various schemes associated with quantum computing have been proposed for quantum information storage and transfers, such as superconducting coplanar waveguide resonators and dual waveguide devices $[1,2]$. A waveguide can be defined as a quantum wire in which the electron wave propagates in quantized modes without losing phase coherence at low temperature. Various proposals have been suggested to implement a semiconductor qubit [3-5]. Among these, an idea is to use parallel quantum waveguides with a coupling-window placed between them [6]. Tuning the window-coupling allows an electron wave interference between the waveguides and can switch the electron motion from one waveguide to the other [7].

The interference in the coupling-region specifies the possible types of qubit-operations: The electron wave can be transferred through either waveguide only (pass gateoperation). It can travel through both waveguides, split equally, implementing the so called square-root-of-Not $(\sqrt{\mathrm{NOT}})$ operation [8]. It can switch totally from one waveguide to the other (Not-operation) [9]. Or it might switch from the first waveguide to the second one and then re-enter the first waveguide (CNot-operation) [10]. There are several parameters and phenomena that can modify the efficiency of the kind of qubit-operation that are mentioned above such as external magnetic field, the Coulomb interaction, electrostatic gate voltage, and the geometry of the coupling window between the waveguides.

Ferry's group proposed magnetically switching transport in an asymmetric double quantum waveguide, in

\footnotetext{
* nra1@hi.is

$\dagger$ vidar@hi.is
}

which an external magnetic field is applied to switch electron motion from one quantum-waveguide to the other [7, 10]. Another suggestion for a switching-qubit is to vary the length of the coupling window (CW) $L_{\mathrm{CW}}$. By varying the length of the $\mathrm{CW}$, a maximum inter-waveguide tunneling can be found that increases the efficiency of the device [7]. Another approach for a switching-qubit is to define a saddle potential in the coupling window instead of a hard-walled well potential. The saddle-potential makes a soft barrier between the two waveguides that washes out resonance peaks, but decreases the efficiency of the qubit [11].

In the present work, we consider a double quantum waveguide (DQW) system with a CW between the waveguides in a static perpendicular magnetic field. Both ends of the DQW are connected to semi-infinite leads with an applied source-drain bias. The coherent electron transport is investigated in the system by using a non-Markovian master equation [12-15]. Smooth Gaussian potentials are used to define the barrier between the waveguides and the saddle-like $\mathrm{CW}$ potential. In addition, two dots are embedded in the waveguides close to the $\mathrm{CW}$ to enhance inter-waveguide transport. We will investigate coherent electron transport in the system by varying the $\mathrm{CW}$ length.

Our results reveal the following conclusions: First, we predict a maximum conductance at resonant energylevels. At a resonance energy, inter-waveguide scattering is enhanced and the charge density splits equally between the waveguides in the absence of the Coulomb interaction as two-electron states are activated in the transport. In this case, the DQW essentially works as a $\sqrt{\mathrm{NOT}}$ operation qubit. In the presence of the Coulomb interaction, the charge current density does not split equally between the waveguides anymore at the resonance since two-electron states are blocked for the bias range used. 
The strength of the blocking can be varied by the geometry or the material of the system. The transient transport through two capacitively coupled parallel quantum dots has been investigated by Moldoveanu et al. in a lattice model for a weaker Coulomb interaction than is assumed here [16]. Second, an external static magnetic field can increase inter-waveguide backward scattering leading to a decrease in the efficiency of the qubit. These results make clear the difference to previous schemes where variable magnetic field was used to control the switching between asymmetric waveguides $[6,11]$. Third, the influence of the side quantum dots (QDs) can be to induce more energy-levels into resonance, and consequently increase the coupling between the waveguides and the interwaveguide transport mechanism. In the presence of side QDs, a Not-operation qubit is realized. The efficiency is slightly decreased in the presence of side QDs because inter-waveguide backward scattering is enhanced. At high magnetic field such that the effective magnetic length is comparable to the radius of the dots, variation of length of the CW does not influence the electron transport characteristics anymore because the electrons are localized in the side dots. Thus, the net charge current is extremely suppressed.

The paper is organized as follows: In Sec. II, we present the model describing the window-coupled double waveguide system based on the quantum master equation (QME) approach. Section III presents our numerical results and discussion. Concluding remarks are addressed in Sec. IV.

\section{MODEL AND THEORY}

In this section, we present a method for calculating the transient ballistic transport in the coupled-window quantum waveguides without and with side QDs. We consider two laterally coupled waveguides connected to two semi-infinite wires from both ends. The lower waveguide (control) and the upper waveguide (target) are coupled through a $\mathrm{CW}$ with length $L_{\mathrm{CW}}$ as is schematically shown in Fig. 1. We consider a control-QD $\left(\mathrm{QD}_{\mathrm{C}}\right)$ and a target-QD $\left(\mathrm{QD}_{\mathrm{T}}\right)$ embedded, respectively, in the controland the target-waveguide near the $\mathrm{CW}$. The total system is exposed to an external magnetic field in the $z$-direction Bẑ. The system is designed such that the electrons are injected from the left lead to only the control-waveguide (red arrow).

Our aim here is to study the charging of, and the output from each of the waveguides by varying the CW length $L_{\mathrm{CW}}$ (blue dashed arrow). In addition, the effects of the Coulomb interaction, the external magnetic field, and the side dots on the conductance will be explored.

The DQW is a two dimensional system with hard-wall confinement in the contact area to the external leads at $x= \pm L_{x} / 2$ with $L_{x}$ the length of the waveguides in the transport direction and parabolic confinement in the $y$ direction. The single-electron Hamiltonian of the DQW

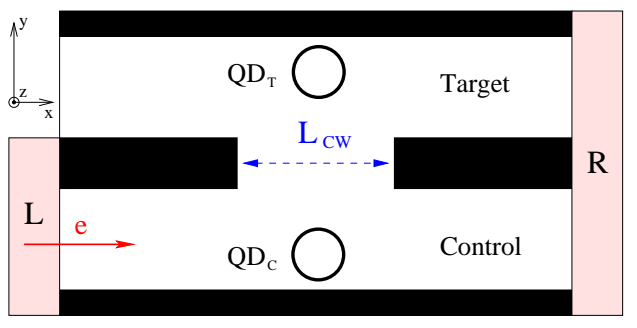

FIG. 1. (Color online) Schematic diagram depicting the double waveguide with a $\mathrm{CW}$ of length $L_{\mathrm{CW}}$ (blue dashed arrow). A quantum dot $\mathrm{QD}_{\mathrm{C}}\left(\mathrm{QD}_{\mathrm{T}}\right)$ is embedded in the control (target) waveguide. An electron from left side enters the control waveguide (red arrow). Asymmetric contacts are indicated by the light red rectangles marked $\mathrm{L}$ and $\mathrm{R}$.

including side QDs in an external magnetic field is

$$
h_{\mathrm{S}}=\frac{\left(\boldsymbol{p}+e \mathbf{A}_{\mathrm{ext}}\right)^{2}}{2 m^{*}}+V_{\mathrm{c}}(x, y)+V_{\mathrm{DW}}(\mathbf{r})+V_{\mathrm{QDs}}(\mathbf{r}),
$$

where $\boldsymbol{p}$ indicates the canonical momentum, $\mathbf{A}_{\text {ext }}=$ $(0,-B y, 0)$ is vector potential of the external magnetic field, and $m^{*}$ is the effective mass of an electron. The confining potential of the DQW is $V_{\mathrm{c}}(x, y)=V_{\mathrm{c}}(x)+V_{\mathrm{c}}(y)$, where $V_{\mathrm{c}}(x)$ stands for a hard-wall confining potential and $V_{\mathrm{c}}(y)=\frac{1}{2} m^{*} \Omega_{0}^{2} y^{2}$ refers to a parabolic confining potential with characteristic energy $\hbar \Omega_{0}$. The Gaussian potential barrier between the two waveguides is presented by $V_{\mathrm{DW}}(\mathbf{r})$ and the side QDs are defined by $V_{\mathrm{QDs}}(\mathbf{r})$. We diagonalize the Hamiltonian of the central system presented in Eq. (1) using a basis [15] built with the eigenfunctions of the first two terms of (1) to find the singleelectron energy spectrum $E_{\mathrm{n}}$, where $n$ is the composite quantum number of a single-electron state.

Below, we shall demonstrate how the DQW is connected to the leads and show the time evolution of electron in the system.

\section{A. DQW connected to leads}

We connect the DQW to two leads that act as electron reservoirs via a contact region. The central system and the leads have the same confinement energy and the same strength of the perpendicular magnetic field. The total Hamiltonian of the system is then

$$
\begin{aligned}
H(t) & =\sum_{n} E_{n} d_{n}^{\dagger} d_{n}+\frac{1}{2} \sum_{i j r s}\left\langle V_{\text {Coul }}\right\rangle d_{i}^{\dagger} d_{j}^{\dagger} d_{s} d_{r} \\
& +\sum_{l=\mathrm{L}, \mathrm{R}} \int d \boldsymbol{q} \epsilon^{l}(\boldsymbol{q}) c_{\boldsymbol{q}^{\dagger}} c^{l} \boldsymbol{q} \\
& +\sum_{l=\mathrm{L}, \mathrm{R}} \chi^{l}(t) \sum_{n} \int d \boldsymbol{q}\left[c^{l} \boldsymbol{q}^{\dagger} T_{\boldsymbol{q}_{n}}^{l} d_{n}+d_{n}^{\dagger}\left(T_{n}^{l} \boldsymbol{q}\right)^{*} c_{\boldsymbol{q}}^{l}\right]
\end{aligned}
$$

The first term describes the central system, where $E_{n}$ is the single-electron energy of state $|n\rangle$ of the central sys- 
tem, and $d_{n}^{\dagger}\left(d_{n}\right)$ denotes the electron creation (annihilation) operator respectively. The second term is the mutual Coulomb interaction between electrons in the central system. The third term of the Hamiltonian denotes the semi-infinite $l^{\text {th }}$ lead, where $l$ refers to the left $\mathrm{L}$ or the right $\mathrm{R}$ lead with the dummy index $\boldsymbol{q}$ representing the "momentum" of an electron in the lead and its subband index $[15], \epsilon^{l}(\boldsymbol{q})$ is the single-electron energy spectrum in lead $l$, and the electron creation and annihilation operator in lead $l$ are $c_{\boldsymbol{q}}^{l}$ and $c_{\boldsymbol{q}}^{l}$, respectively.

The last term is the time-dependent coupling Hamiltonian that connects the central system, the DQW to the external leads via a coupling tensor $T_{\boldsymbol{q}_{n}}^{l}$, and $\chi^{l}(t)$ dictates the time-dependence of the coupling. The coupling Hamiltonian describes the transfer of an electron between the single-electron state of the central system $|n\rangle$ and the single-electron state of the leads $|\boldsymbol{q}\rangle$

$$
T_{\boldsymbol{q}_{n}}^{l}=\int d \mathbf{r} d \mathbf{r}^{\prime} \psi_{\boldsymbol{q}^{l}}^{l}\left(\mathbf{r}^{\prime}\right)^{*} g_{\boldsymbol{q}_{n}}^{l}\left(\mathbf{r}, \mathbf{r}^{\prime}\right) \psi_{n}^{\mathrm{S}}(\mathbf{r})
$$

$\psi_{n}^{\mathrm{S}}(\mathbf{r})$ and $\psi_{\boldsymbol{q}}^{l}\left(\mathbf{r}^{\prime}\right)$ are the corresponding single-electron wave functions of the DQW system and the leads. $g_{\boldsymbol{q}_{n}}^{l}\left(\mathbf{r}, \mathbf{r}^{\prime}\right)$ is a nonlocal coupling kernel

$$
\begin{aligned}
g_{\boldsymbol{q} n}^{l}\left(\mathbf{r}, \mathbf{r}^{\prime}\right) & =g_{0}^{l} \exp \left[-\delta_{x}^{l}\left(x-x^{\prime}\right)^{2}-\delta_{y}^{l}\left(y-y^{\prime}-\alpha\right)^{2}\right] \\
& \times \exp \left(-\Delta_{n}^{l}(\boldsymbol{q}) / \Delta\right),
\end{aligned}
$$

where $g_{0}$ is the coupling strength, $\delta_{x}^{l}$ and $\delta_{y}^{l}$ are the coupling range parameters in the $x$ - and $y$-direction, respectively, $\Delta$ controls together with $\Delta_{n}^{l}(\boldsymbol{q})=\left|E_{n}-\epsilon^{l}(\boldsymbol{q})\right|$ the energy affinity between states in the leads and the central system [13], and $\alpha$ is a skewing parameter that shifts the weight of the coupling to the lower waveguide for an appropriate value stated below.

\section{B. QME and Charge current density}

In this subsection, we present how the time evolution of the open system is calculated in order to study time-dependent transport properties within the QME approach [17]. The time evolution of our system obeys the quantum Liouville-von Neumann equation

$$
\frac{d W(t)}{d t}=-\frac{i}{\hbar}[H(t), W(t)]
$$

where $W(t)$ is the density operator of the total system.

In order to focus our attention on the open central system we introduce a reduced density operator (RDO) with respect to it, by taking the trace over the Fock space with respect to the leads $\rho(t)=\operatorname{Tr}_{\mathrm{L}} \operatorname{Tr}_{\mathrm{R}} W(t)$ with the initial condition $W\left(t<t_{0}\right)=\rho_{\mathrm{L}} \rho_{\mathrm{R}} \rho_{\mathrm{S}}$, where $\rho_{\mathrm{L}}$ and $\rho_{\mathrm{R}}$ are the density operators of the isolated left and the right leads, respectively. The density operator of the $l^{\text {th }}$ lead can be defined as $\rho_{l}=e^{-\beta\left(H_{l}-\mu_{l} N_{l}\right)} / \operatorname{Tr}_{l}\left\{e^{-\beta\left(H_{l}-\mu_{l} N_{l}\right)}\right\}$, where $\beta=1 /\left(k_{B} T\right)$ and $k_{B}$ is the Boltzmann constant, and $N_{l}$ is the number of electrons in the leads. The density operator of the central system at $t=t_{0}$ being $\rho\left(t_{0}\right)=\rho_{\mathrm{S}}[18]$ allows us to obtain the equation of motion for the RDO in the following form [19]

$$
\frac{d \rho(t)}{d t}=-i \mathcal{L}_{\mathrm{S}} \rho(t)+\int_{t_{0}}^{t} d t^{\prime} \mathcal{K}\left(t, t^{\prime}\right) \rho\left(t^{\prime}\right),
$$

where $\mathcal{L}_{\mathrm{S}} \cdot=\left[H_{S}, \cdot\right] / \hbar$ is the Liouvillian with respect to the time-independent Hamiltonian $H_{S}$ of the central system and $\mathcal{K}\left(t, t^{\prime}\right)$ is an integral kernel with its roots in the dissipative time-dependent coupling to the leads $[15,19]$.

In order to explore the switching processes between the waveguides, we define the net charge current as

$$
I_{\mathrm{Q}}(t)=I_{\mathrm{L}}(t)-I_{\mathrm{R}}(t)
$$

where $I_{\mathrm{L}}(t)$ refers to the partial current from the left lead into the control-waveguide and $I_{\mathrm{R}}(t)$ indicates the partial current into the right lead from both waveguides [20]. In Eq. (7) the negative sign in front of $I_{\mathrm{R}}$ is since a positive charge current is defined as the current into the central system, but the currents $I_{L}$ and $I_{R}$ are defined positive from left to right.

To monitor the dynamic evolution of the electrons in the central system, we calculate the expectation value of charge current density operator

$$
\mathbf{J}(\mathbf{r}, t)=\operatorname{Tr}(\hat{\rho}(t) \hat{\mathbf{J}}(\mathbf{r}))
$$

where the charge current density operator is defined by

$$
\begin{aligned}
\hat{\mathbf{J}}(\mathbf{r}) & =\sum_{n n^{\prime}}\left(\frac{e \hbar}{2 m^{*} i}\left[\psi_{n}^{\mathrm{S} *}(\mathbf{r})\left(\nabla \psi_{n^{\prime}}^{\mathrm{S}}(\mathbf{r})\right)-\left(\nabla \psi_{n}^{\mathrm{S} *}(\mathbf{r})\right) \psi_{n^{\prime}}^{\mathrm{S}}(\mathbf{r})\right]\right. \\
& \left.+\frac{e^{2}}{m^{*}} \mathbf{A}_{\mathrm{ext}}(\mathbf{r}) \psi_{n}^{\mathrm{S} *}(\mathbf{r}) \psi_{n^{\prime}}^{\mathrm{S}}(\mathbf{r})\right) d_{n}^{\dagger} d_{n^{\prime}}
\end{aligned}
$$

In the following, we shall demonstrate the influence of the length of the CW, external magnetic field, and the side QDs on the coherent electron transport through the system and the charge current density in the central system in order to investigate inter-waveguide forward and backward scattering processes.

\section{RESULTS}

In this section, we shall discuss our numerical results of the ballistic transport properties through the double waveguide system made of a GaAs semiconductor with the electron effective mass $m^{*}=0.067 m_{e}$. The length of the central system is $L_{x}=300 \mathrm{~nm}$ which is assumed to be much smaller than the phase coherent length $L_{\phi}$. At low temperature $T \sim 0.1-2.0 \mathrm{~K}$, the phase coherent length of semiconductor based ( $\mathrm{GaAs} / \mathrm{Al}_{1-\mathrm{x}} \mathrm{Ga}_{\mathrm{x}} \mathrm{As}$ ) electron waveguide is $\sim(30-40) \times 10^{3} \mathrm{~nm}[21]$. The coherence in the electron transport in the double waveguide 
is an essential requirement for constructing a qubit. For the system placed in a static or vanishing external magnetic field, its length scales can be characterized by the effective magnetic length $a_{w}=\left\{\hbar /\left(m^{*} \sqrt{\left(\omega_{c}^{2}+\Omega_{0}^{2}\right)}\right)\right\}^{1 / 2}$, where the cyclotron frequency $\omega_{c}=e B / m^{*}$ and the transverse confinement energy is $\hbar \Omega_{0}=1.0 \mathrm{meV}$. Numerically the effective magnetic length can be expressed as

$$
\begin{aligned}
a_{w} & =\left(\frac{\hbar}{m^{*} \Omega_{0}}\right)^{1 / 2}\left(\frac{1}{1+\left(e B /\left(m^{*} \Omega_{0}\right)\right)^{2}}\right)^{1 / 4} \\
& =\frac{33.74}{\sqrt[4]{1+2.982[B(\mathrm{~T})]^{2}}} \mathrm{~nm}
\end{aligned}
$$

For the case of a vanishing magnetic field we assign to it a finite very small value in order to avoid numerical problems caused by exact degeneration of states with opposite spin.

We assume the central system is connected to the external leads acting as electron reservoirs with chemical potential in the left (right) lead $\mu_{L}=4.0 \mathrm{meV}$ $\left(\mu_{R}=3.0 \mathrm{meV}\right)$, implying an applied potential difference or bias window $\Delta \mu=e V_{\text {bias }}=1.0 \mathrm{meV}$. Furthermore, the temperature of the leads is fixed at $\mathrm{T}=0.5 \mathrm{~K}$.

The potential of the double quantum waveguides is shown in Fig. 2 with the side QDs located near the CW in order to influence the inter-waveguide transport. In

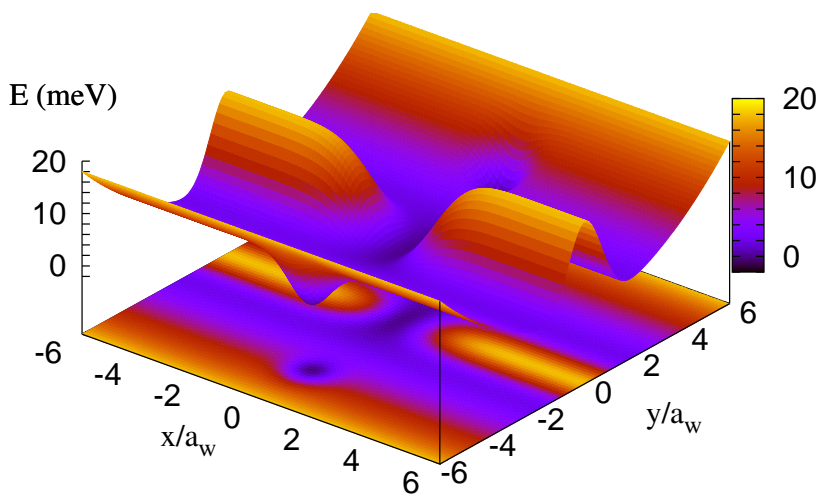

FIG. 2. (Color online) The potential (in meV) defining the DQW central system with the CW and the side QDs. The parameters are $\hbar \Omega_{0}=1.0 \mathrm{meV}, B=0.0 \mathrm{~T}, a_{w}=33.72 \mathrm{~nm}$, $L_{\mathrm{CW}}=100 \mathrm{~nm}$, and $R_{\mathrm{Dot}}=25 \mathrm{~nm}$.

the following, we shall explore the influence of the CW and the side QDs on the charging of and the transport through the system.

\section{A. DQW without side QDs}

We start by considering an ideal window-coupled DQW without side QDs. The injected electrons are assumed to come from the left lead only into the controlwaveguide. This asymmetry in the injection is accomplished with the choice of the skewing parameter $\alpha=$
$4.0 a_{w}$ defined in Eq. (4) and only used for the left lead. In addition, we assume the two quantum waveguides to be of the same width. They are separated by a Gaussian potential barrier, in which the control- and targetwaveguide interact with each other via a CW. The DQW system is described by

$$
V_{\mathrm{DQW}}(\mathbf{r})=V_{\mathrm{B}}(y)+V_{\mathrm{CW}}(x, y),
$$

where $V_{\mathrm{B}}(y)$ is the barrier potential between the quantum waveguides defined as

$$
V_{\mathrm{B}}(y)=V_{0} \exp \left(-\beta_{0}^{2} y^{2}\right)
$$

with $V_{0}=18.0 \mathrm{meV}$ and $\beta_{0}=0.03 \mathrm{~nm}^{-1}$, as well as a $\mathrm{CW}$ potential

$$
V_{\mathrm{CW}}(x, y)=-V_{0} \exp \left(-\beta_{x}^{2} x^{2}-\beta_{y}^{2} y^{2}\right)
$$

with $V_{0}=18.0 \mathrm{meV}$, and $\beta_{y}=0.03 \mathrm{~nm}^{-1}$ implying a barrier width $W_{\mathrm{B}} \simeq 66.5 \mathrm{~nm}$ for the first subband which can prevent electron tunneling between the waveguides through the barrier. $\beta_{x}$ is a parameter that determines the CW length. Thus, the length of the CW can be defined as $L_{\mathrm{CW}}=2 / \beta_{x}$.

We begin to analyze the results by presenting Fig. 3 which shows the net charge current $I_{\mathrm{Q}}$ as a function of the CW length $L_{\mathrm{CW}}$ at $t=200$ ps and $B=0.0$ T for noninteracting electrons (solid blue) and for Coulomb interacting electrons (dashed red). The Coulomb interaction reduces the overall current slightly.

The oscillations in the net charge current are indicative of a possible charge transport between the controlwaveguide and the target-waveguide. The oscillations give rise to a peak and a dip in the net charge current at $L_{\mathrm{CW}} \simeq 40$ and $110 \mathrm{~nm}$, respectively. Similar oscillation features have been found by Zibold et al. and Gong et al. in the absence of the Coulomb interaction, and put in relation to energy-dependent inter-waveguide transmission in the quantum regime. They pointed out that the inter-waveguide transmission can be enhanced when the energy levels of the coupled-waveguide system achieve a resonance conditions for a specific CW length [22, 23].

To demonstrate the resonant energy levels in our system, we present Fig. 4 which shows the many-electron (ME) energy spectra for the DQW system as a function of the $\mathrm{CW}$ length at $B=0.0 \mathrm{~T}$ including one-electron states (1ES, red dots) and two-electron states (2ES, blue dots). The ME spectrum without the mutual Coulomb interaction is displayed in Fig. 4(a), but the interacting spectrum in Fig. 4(b). The only difference between the energy spectra is that the Coulomb interaction has raised most of the $2 \mathrm{ES}$ well above the bias window in Fig. 4(b). The 2ES do not contribute to the electron transport in the presence of the Coulomb interaction because they are far from the chosen bias window, leading to a decrease in the net charge current.

In the case of no coupling window the two waveguides are only coupled through tunneling and the Coulomb interaction [16] leading to nearly degenerate states. When 


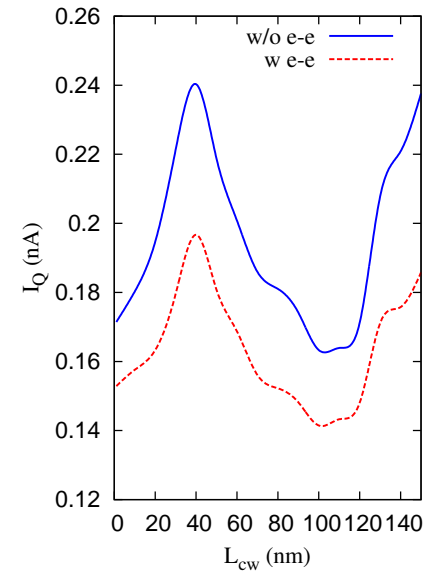

FIG. 3. (Color online) The net charge current $I_{\mathrm{Q}}$ versus CW length $L_{\mathrm{CW}}$ without (w/o) (blue solid) and with (w) (red dashed) electron-electron (e-e) interaction for magnetic field $B=0.0 \mathrm{~T}$ The chemical potentials are $\mu_{L}=4.0 \mathrm{meV}$ and $\mu_{R}=3.0 \mathrm{meV}$ implying $\Delta \mu=1.0 \mathrm{meV}$.

the length of the CW $L_{\mathrm{CW}}$ is increased we thus find: First, the energy spectrum decreases monotonically [22]. Second, the near degeneration of the energy-levels is lifted. As a result, this leads to a crossover of energy levels (resonance) between the control- and targetwaveguide at $L_{\mathrm{CW}} \simeq 40 \mathrm{~nm}$ (left green rectangular) which corresponds to the current-peak in Fig. 3. The resonance between the waveguides enhances the interwaveguide electron transport and increases the net charge current. The splitting of the energy levels increases at a higher window-coupling length such as $L_{\mathrm{CW}} \simeq 110 \mathrm{~nm}$ (right green rectangular) indicating weaker resonances and more back-scattering inter-waveguide transport.

To further explain the peak and the dip in the net charge current, we present the charge current density at $B=0.0 \mathrm{~T}$ and $t=200 \mathrm{ps}$ in Fig. 5 for the noninteracting system (left panels) and the interacting one (right panels) for the peak and the dip net charge current at $L_{\mathrm{CW}} \simeq$ $40 \mathrm{~nm}$ (a-b) and $110 \mathrm{~nm}$ (c-d), respectively, shown in Fig. 3.

The dynamical evolution occurring in the CW implements different types of quantum logic gates in our system. In Fig. 5(a) the charge current density in the absence of the Coulomb interaction reveals the following electron motion in the waveguides at $L_{\mathrm{CW}} \simeq 40 \mathrm{~nm}$ : The charge is injected into the control input, then it exits equally from both control and target output. In the DQW the charge exhibits partial inter-waveguide forward scattering which is similar to the condition of having equal electron transmission ratio in the double waveguides at a resonance [22]. The DQW here can be defined as a beam splitter or a $\sqrt{\mathrm{NOT}}$-operation quantum logic gate $(\sqrt{\text { NOT}}$-operation qubit) $[8]$.

Figure $5(\mathrm{c})$ shows the charge current density in the current-dip at $L_{\mathrm{CW}} \simeq 110 \mathrm{~nm}$. The charge current den-
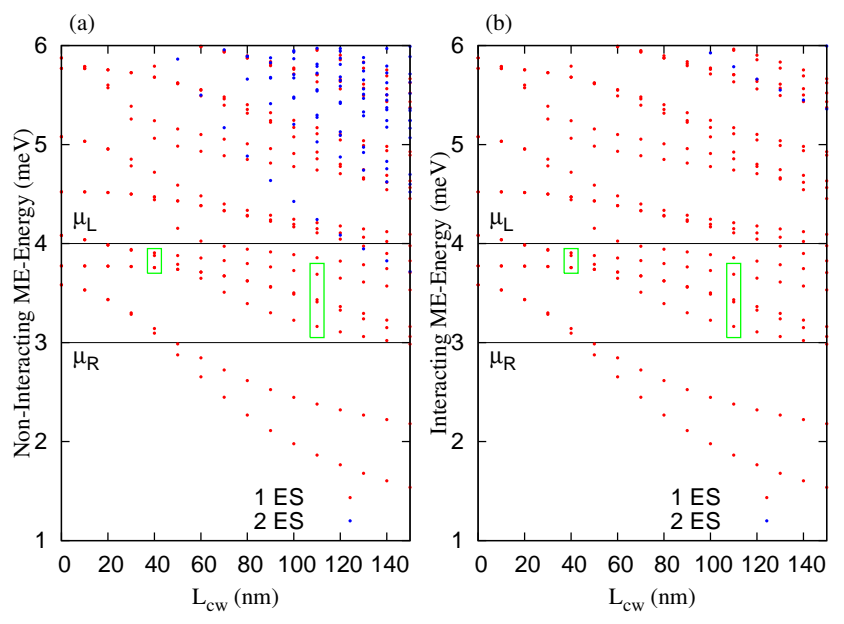

FIG. 4. (Color online) ME energy spectrum in the central system as a function of CW length $L_{\mathrm{CW}}$ without (a), and with (b) the electron-electron Coulomb interaction including one-electron states (1ES, red dots) and two-electron states (2ES, blue dots) at $B=0.0 \mathrm{~T}$. The one-electron states in the left green rectangle are close to be in resonance, but the one-electron states in the right green rectangle are not. The chemical potentials are $\mu_{L}=4.0 \mathrm{meV}$ and $\mu_{R}=3.0 \mathrm{meV}$ (black) implying $\Delta \mu=1.0 \mathrm{meV}$.

sity switches from the control- to the target-waveguide with a small ratio of inter-waveguide backward scattering. The charge switching property of the system here can be introduced as a Not-operation quantum logic gate [11]. The net charge current is suppressed here due to the presence of an inter-waveguide backward scattering that forms the current-dip. The cause of the interwaveguide backward scattering is the geometry of the system, the symmetric waveguides, while in the asymmetric system the back scattering can be avoided [6].

With the Coulomb interaction the charge current density is very similar for the dip near $110 \mathrm{~nm}$ (comparing Fig's 5(c) and (d)), but different for the peak at 40 $\mathrm{nm}$, where very little scattering into the target waveguide is seen (comparing Fig. 5(a) and (b)). Without the Coulomb interaction 2ES contribute up to $1 / 3$ of the charge current, but the interaction reduces this with Coulomb blocking to a negligible quantity. The forward inter-waveguide scattering in Fig. 5(a) is facilitated by two-electron processes and states. In the present system the Coulomb interaction blocks these processes to a large extent.

The effects of a static external magnetic field are presented in Fig. 6(a) which shows the net charge current $I_{\mathrm{Q}}$ as a function of the $\mathrm{CW}$ length $L_{\mathrm{CW}}$ at $t=200 \mathrm{ps}$ in the presence of the Coulomb interaction for different values of the magnetic field $B=0.0 \mathrm{~T}$ (blue solid), $0.1 \mathrm{~T}$ (green dashed), and $0.2 \mathrm{~T}$ (red dashed). For comparison we display in Fig. 6(b) the left and the right currents $I_{\mathrm{L}}$ and $I_{\mathrm{R}}$ at $B=0.0 \mathrm{~T}$ and the same point in time. The comparison shows that at $t=200$ ps the system is still in 
(a)

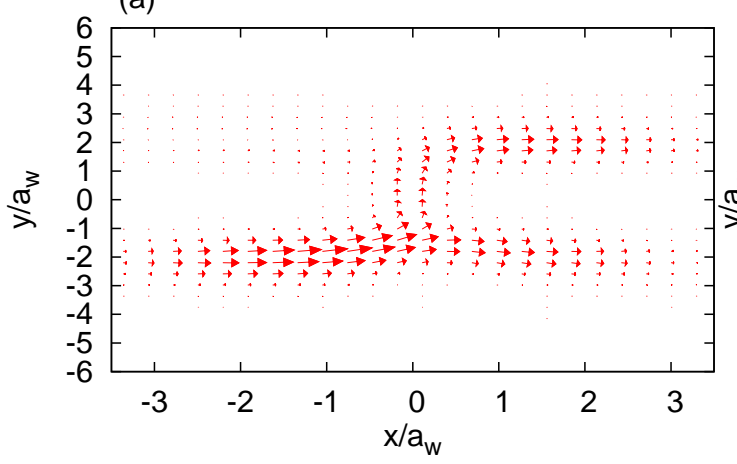

(c)

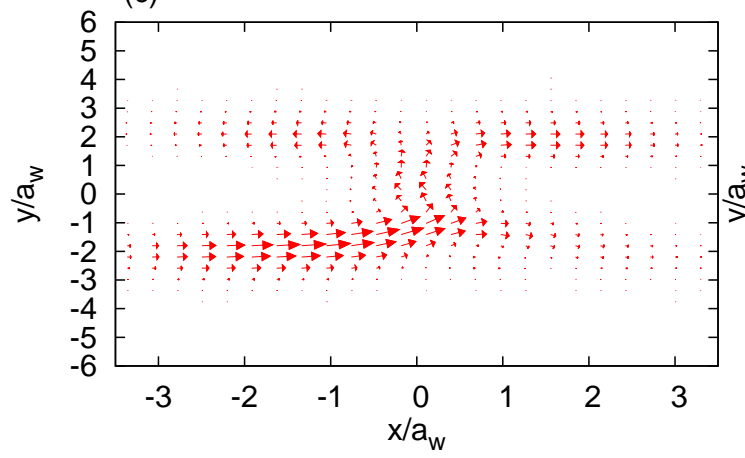

(b)

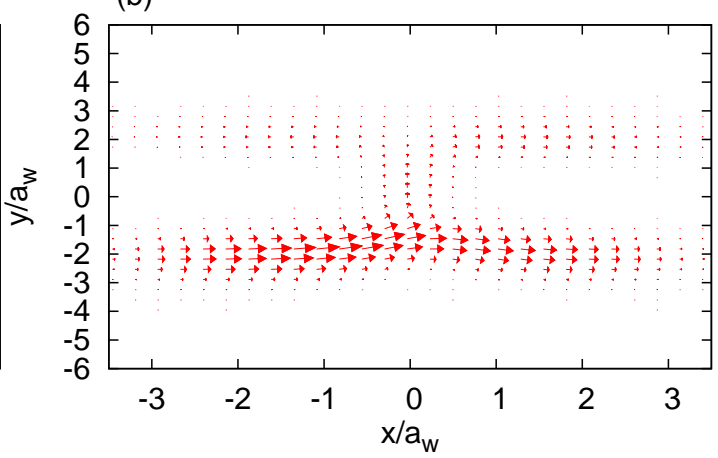

(d)

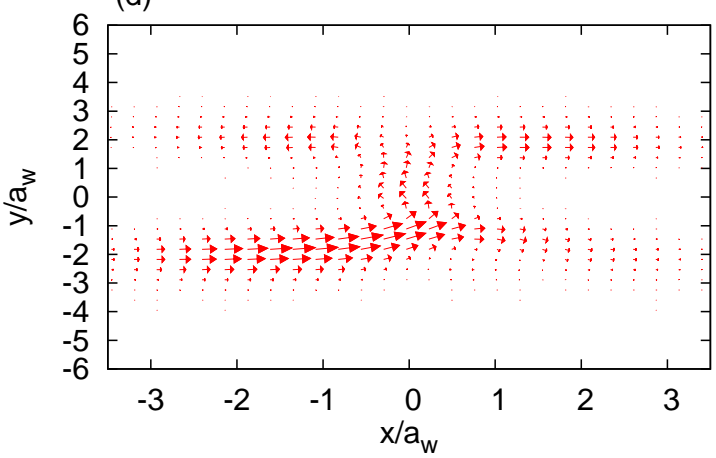

FIG. 5. (Color online) Charge current density in the DQW at $t=200$ ps without (left panels) with (right panels) electronelectron Coulomb interaction in the peak and the dip net charge current at $L_{\mathrm{CW}} \simeq 40 \mathrm{~nm}$ (a)-(b) and $110 \mathrm{~nm}$ (c)-(d), respectively, shown in Fig. 3 in the case of $B=0.0 \mathrm{~T}$ (blue solid). The effective magnetic length is $a_{w}=33.72 \mathrm{~nm}$

the charging phase, but the overall shape of the currents is similar.

The role of the external magnetic field in our system is different from the role it plays in many models where researchers have applied a variable magnetic field to switch between different processes in the waveguides. The external magnetic field has been used to switch an electron current from one quantum-waveguide to the other one in a window coupled double waveguide [6]. In our model, we observe switching characteristic in absence of a magnetic field $B=0.0 \mathrm{~T}$. In the presence of stronger magnetic field, the electron motion is affected by the Lorentz force in which the electrons tend to a circular motion in both control- and target-waveguides increasing the electron dwell-time in the system. The inter-waveguide backward scattering is enhanced while a suppression in the inter-waveguide forward scattering is observed in the presence of strong magnetic field. Therefore, the net charge current decreases as shown in Fig. 6(a) in the case of $B=0.1 \mathrm{~T}$ (green dashed) and $0.2 \mathrm{~T}$ (red dotted).

To understand the reasons for the suppression of the net charge current in the presence of a higher magnetic field, we show in Fig. 7 the charge current density at magnetic field $B=0.2 \mathrm{~T}$ in the peak at $L_{\mathrm{CW}} \simeq 40 \mathrm{~nm}$ (a) and the dip at $L_{\mathrm{CW}} \simeq 110 \mathrm{~nm}$ (b) of the net charge current shown in Fig. 6(a) (red dotted). An obvious explanation is: The perpendicular magnetic field reduces
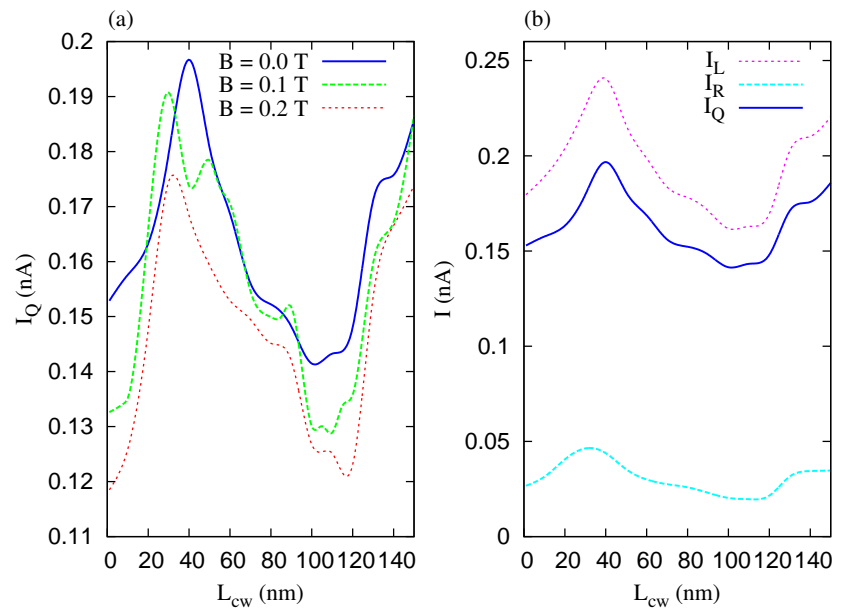

FIG. 6. (Color online) (a) The net charge current $I_{\mathrm{Q}}$ versus $\mathrm{CW}$ length $L_{\mathrm{CW}}$ in the presence of the Coulomb interaction for different values of the magnetic field $B=0.0 \mathrm{~T}$ (blue solid), $0.1 \mathrm{~T}$ (green dashed), and $0.2 \mathrm{~T}$ (red dotted) at time $t=200 \mathrm{ps}$. (b) The current from the left lead $I_{\mathrm{L}}$ (pink dotted), the current into the right lead $I_{\mathrm{R}}$ (blue dashed), and the net charge current $I_{\mathrm{Q}}$ (blue solid) for $B=0.0 \mathrm{~T}$ versus the $\mathrm{CW}$ length. The chemical potentials are $\mu_{L}=4.0 \mathrm{meV}$ and $\mu_{R}=3.0 \mathrm{meV}$ implying $\Delta \mu=1.0 \mathrm{meV}$. 
(a)

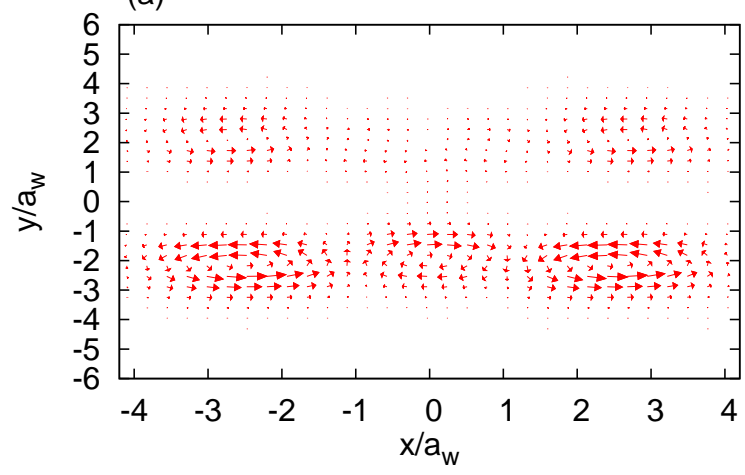

(b)

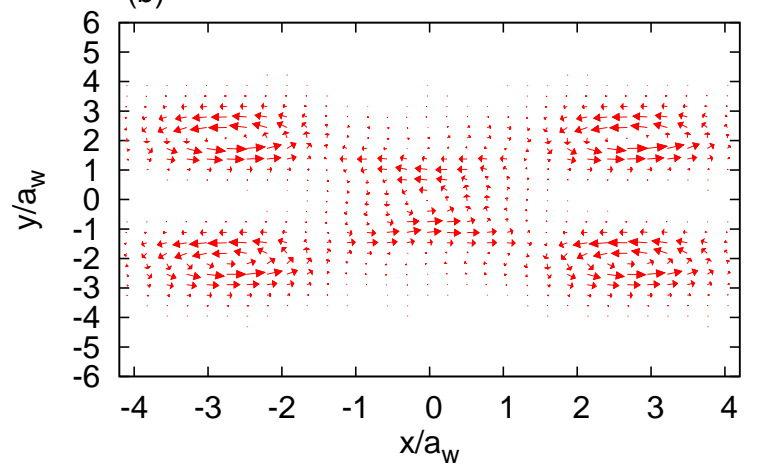

FIG. 7. (Color online) Charge current density in the DQW at $t=200 \mathrm{ps}$ in the presence of the Coulomb interaction in the peak at $L_{\mathrm{CW}} \simeq 40 \mathrm{~nm}(\mathrm{a})$, and the dip at $L_{\mathrm{CW}} \simeq 110 \mathrm{~nm}$ (b) net charge current of Fig. 6(a) in the case of $B=0.2 \mathrm{~T}$ (red dotted). The effective magnetic length is $a_{w}=32.76 \mathrm{~nm}$.

the effective barrier height and increases the splitting of the energy levels at the crossover energy or resonance energy (not shown). Therefore the charge current density does not equally split between the waveguides or localize in only control-waveguide, but rather an inter-waveguide backward scattering is observed due to the Lorentz force, consequently the net charge current is suppressed and we observe edge states forming in each of the waveguides. In this case the double-waveguide system does neither play the role of a $\sqrt{\mathrm{NOT}}$-operation nor a Not-operation qubit in the presence of a higher magnetic field.

In the following, we shall study the influence of the side QDs in the DQW for different values of the magnetic field.

\section{B. DQW with side QDs}

In this section, we consider a side dot embedded in each waveguide to enhance the inter-waveguide transport as is shown in Fig. 2. The dots to the sides of the CW may be expected to increase the dwell time in the coupling region and change the resonance condition between the two waveguides. The window-coupling DQW potential with side QDs is described by

$$
V_{\mathrm{DQW}}(\mathbf{r})=V_{\mathrm{B}}(y)+V_{\mathrm{CW}}(x, y)+V_{\mathrm{Dot}}(x, y),
$$

where $V_{\text {Dot }}(x, y)$ is the side dot potential that is defined as

$$
V_{\text {Dot }}(x, y)=-V_{\mathrm{D}} \exp \left(-\gamma_{x}^{2} x^{2}-\gamma_{y}^{2}\left(y-y_{0}\right)^{2}\right),
$$

with $V_{\mathrm{D}}=8.0 \mathrm{meV}, y_{0}=5.6 \mathrm{a}_{w}$, and $\gamma_{\mathrm{x}}=\gamma_{\mathrm{y}}=0.04$ $\mathrm{nm}^{-1}$ implying the radius of each side-dot $R_{\text {Dot }} \approx 25 \mathrm{~nm}$.

Figure 8(a) shows the net charge current as a function of the $\mathrm{CW}$ length at $t=200 \mathrm{ps}$ in the presence of the Coulomb interaction for different values of the magnetic field $B=0.0 \mathrm{~T}$ (blue solid), $0.1 \mathrm{~T}$ (green dashed), and $0.2 \mathrm{~T}$ (red dashed). An oscillation in the net charge current is again established in a vanishing magnetic field $0.0 \mathrm{~T}$, while at higher magnetic field values 0.1 and $0.2 \mathrm{~T}$ some extra fluctuations in the current oscillation characteristic are observed. The existence of fluctuations correlates with dynamic motion of the charge in the system in which electrons participate in an inter-waveguide backward or forward scattering at different $\mathrm{CW}$ length. If the magnetic field is increased to $B=0.9 \mathrm{~T}$, such that the effective magnetic length is close to the dot radius $a_{w}=R_{\text {Dot }}$ the net charge current is suppressed to vanishing values (not shown). In that case, varying of the $\mathrm{CW}$ length does not affect the electron transport in the system. There are several reasons that lead to the almost vanishing net charge current at high magnetic field such as edge effects, inter-waveguide backward scattering and localized electrons in the dots as the effective magnetic length is close to the dot radius.

We present Fig. 8(b) to compare the left partial current (pink dotted), the right partial current (blue dashed) to the net charge current (blue solid) at $B=0.0 \mathrm{~T}$. It can be seen that the partial currents have the same oscillation features and develop a peak and a current dip at the same CW lengths.

In order to understand the characteristics of net charge current, we refer to the energy spectrum and the charge current density. Figure 9 shows the ME-energy versus the window-coupling length at $B=0.0 \mathrm{~T}$ including oneelectron states (1ES, red dots) and two electron states (2ES, blue dots). It should be noted that more energy levels get into resonance at $L_{\mathrm{CW}} \simeq 40 \mathrm{~nm}$ (left green rectangular) in the presence of the side-gate dots which indicates a stronger coupling between the controland the target-waveguide. At larger $\mathrm{CW}$ length such as $L_{\mathrm{CW}} \simeq 110 \mathrm{~nm}$ (right green rectangular), the energy splitting increases resulting in different electron motion in the system.

The electron motion in the waveguides is completely changed in the presence of the side QDs. Figure 10 displays the charge current density at $B=0.0 \mathrm{~T}$ and $L_{\mathrm{CW}} \simeq 40 \mathrm{~nm}$ (a) and current-dip at $L_{\mathrm{CW}} \simeq 110 \mathrm{~nm}$ (b) shown in Fig. 8(a) (blue solid). In Fig. 10(a) the charge current density is displayed for $L_{\mathrm{CW}} \simeq 40 \mathrm{~nm}$. The 

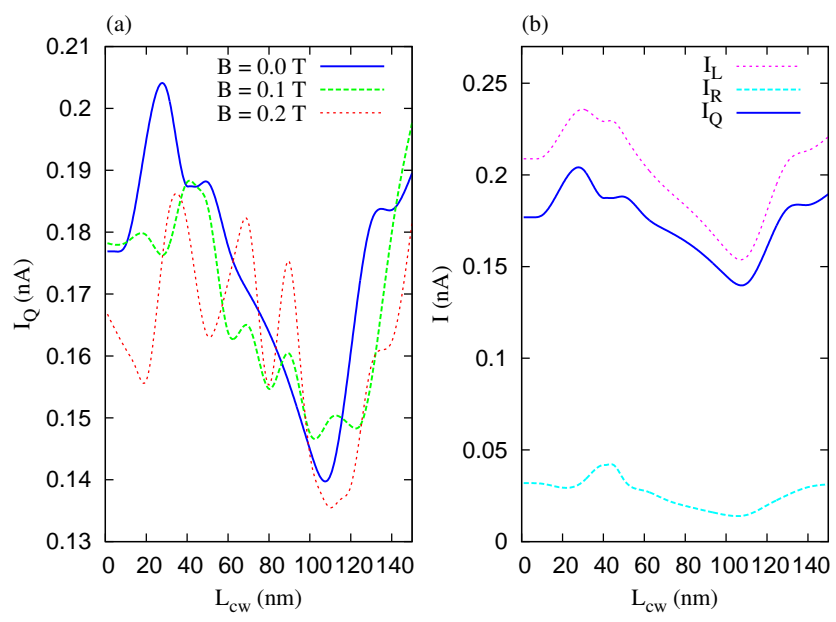

FIG. 8. (Color online) The net charge current $I_{\mathrm{Q}}$ versus the window-coupling length $L_{\mathrm{CW}}$ in the presence of the Coulomb interaction for different magnetic field $B=0.0 \mathrm{~T}$ (blue solid), $0.1 \mathrm{~T}$ (green dashed), $0.2 \mathrm{~T}$ (red dotted) ,0.9 T (pink dotted) at time $t=200 \mathrm{ps}$. (b) The current from the left lead $I_{\mathrm{L}}$ (pink dotted), the current into the right lead $I_{\mathrm{R}}$ (blue dashed), and the net charge current $I_{\mathrm{Q}}$ (blue solid) for $B=0.0 \mathrm{~T}$ versus the CW length. The chemical potentials are $\mu_{L}=4.0 \mathrm{meV}$ and $\mu_{R}=3.0 \mathrm{meV}$ implying $\Delta \mu=1.0 \mathrm{meV}$.

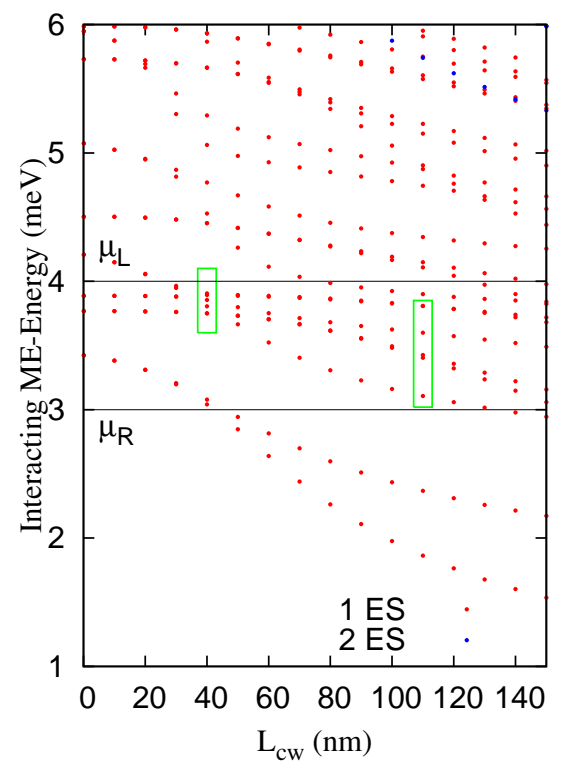

FIG. 9. (Color online) The interacting ME energy spectrum of the central system with side QDs as a function of the CW length $L_{\mathrm{CW}}$ including one-electron states (1ES, red dots) and two electron states ( $2 \mathrm{ES}$, blue dots) at $B=0.0 \mathrm{~T}$. The oneelectron states in the left green rectangular are close to be in resonance, but the one-electron states in the right green rectangular are not. The chemical potentials are $\mu_{L}=4.0 \mathrm{meV}$ and $\mu_{R}=3.0 \mathrm{meV}$ (black) implying $\Delta \mu=1.0 \mathrm{meV}$. charge in the control-waveguide is switched to the targetwaveguide, thus a Not-operation is realized. Comparing to the system without side-gate dots in Fig. 5(b), the inter-waveguide transport is enhanced due to a stronger coupling between the waveguides at the resonant-energy levels at $L_{\mathrm{CW}} \simeq 40 \mathrm{~nm}$. Therefore, the side QDs play an essential role to convert the qubit operation in the system. In addition, the inter-waveguide backward scattering here reduces the efficiency of the qubit. As we mention previously, the existence of inter-waveguide backward scattering might be due to symmetric waveguides while the backward-scattering in an asymmetric waveguides can be avoided or reduced [24]. Figure 10(b) shows the charge current density at the current-dip $L_{\mathrm{CW}} \simeq 110 \mathrm{~nm}$. The incoming charge into the controlwaveguide encounters the control-QD leading to electron back-scattering in the control-waveguide, thus the net charge current is suppressed forming a current-dip.

We can say that the side-dots play a scattering role in the waveguides in such way that the electrons partially get forward- and backward-scattered in and into both waveguides.

(a)

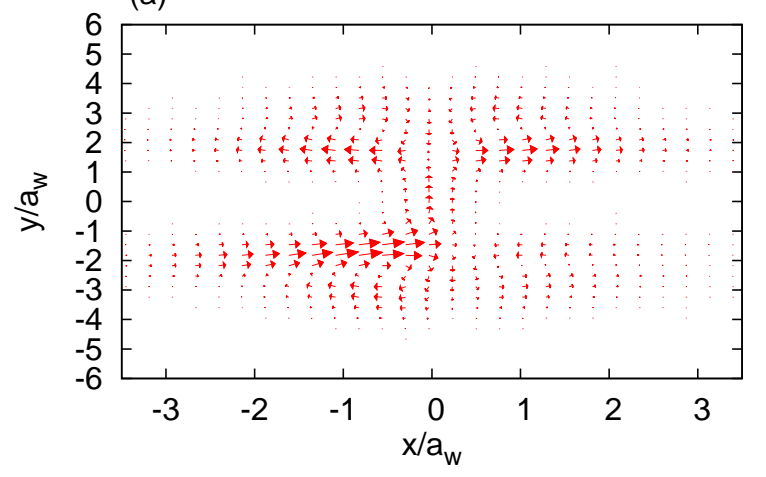

(b)

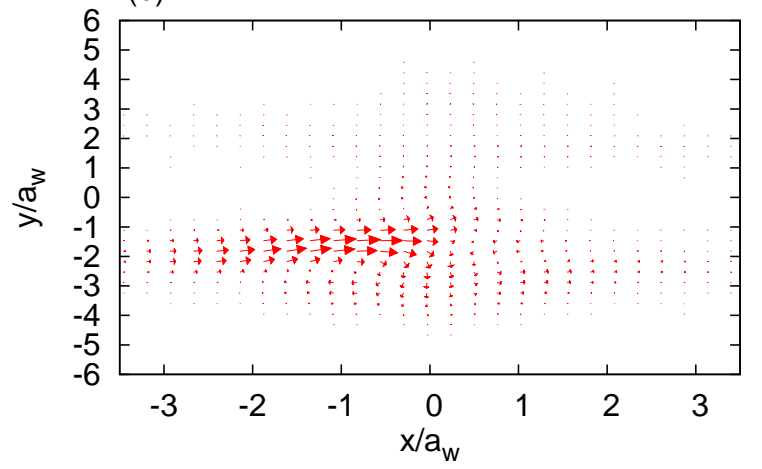

FIG. 10. (Color online) Charge current density in the DQW at $t=200 \mathrm{ps}$ in the presence of the Coulomb interaction in the peak $L_{\mathrm{CW}} \simeq 40 \mathrm{~nm}(\mathrm{a})$, and the dip $L_{\mathrm{CW}} \simeq 110 \mathrm{~nm}(\mathrm{~b})$ of the net charge current of Fig. 8 (a) in the case of $B=0.0 \mathrm{~T}$. Other parameters are $a_{w}=33.72 \mathrm{~nm}$, and $R_{\text {Dot }} \approx 25 \mathrm{~nm}$.

By tuning the external magnetic field to a higher value, 
$B=0.2 \mathrm{~T}$, the electrons manifest different dynamical motion in the waveguides. In Fig. 11 the charge current density is shown in this stronger magnetic field $B=0.2 \mathrm{~T}$ at the $\mathrm{CW}$ length $L_{\mathrm{CW}} \simeq 40$ (a) and $110 \mathrm{~nm}$ (b). The graphs show circular motion, edge states, in each waveguide.

In Fig. 11(a) is shown the current charge density at $L_{\mathrm{CW}} \simeq 40 \mathrm{~nm}$, the electrons in the control-waveguide do not totally switch to the target-waveguide, because of increased energy level splitting at the resonance-energy in the presence of higher magnetic field. In addition, charge is partially localized in the control-QD as the effective magnetic length approaches the radius of the quantum $\operatorname{dot} a_{w} \simeq R_{\text {Dot }}$.
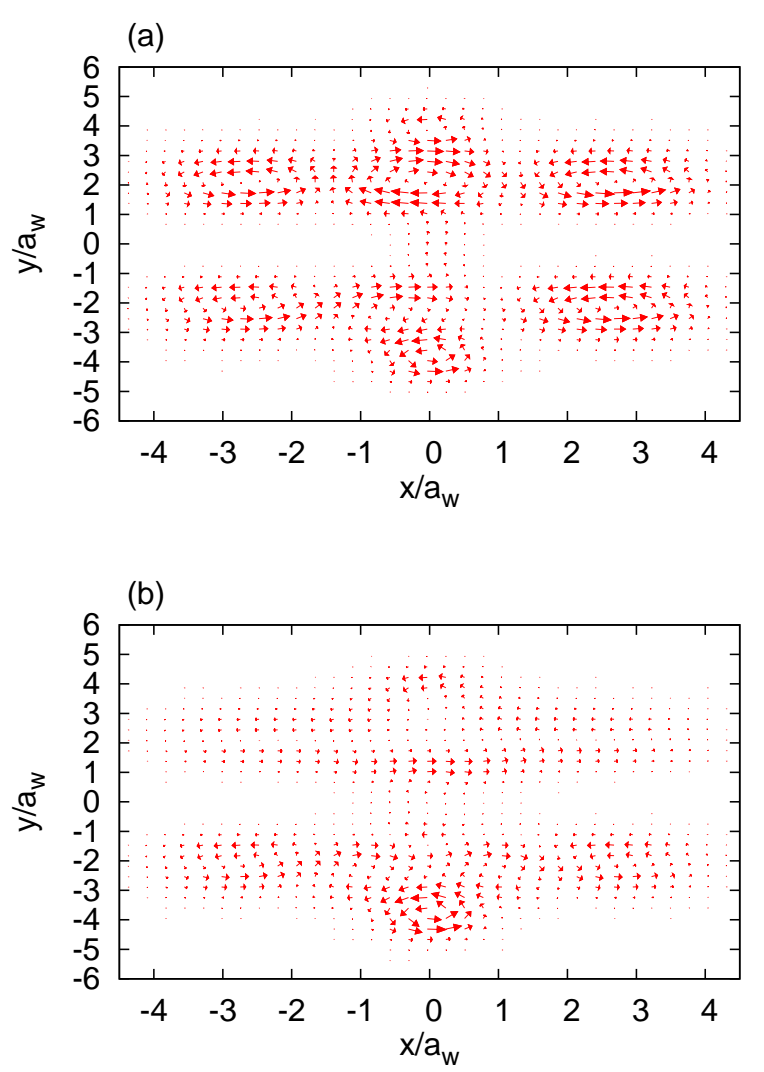

FIG. 11. (Color online) Charge current density in the DQW at $t=200 \mathrm{ps}$ in the presence of the Coulomb interaction in the peak $L_{\mathrm{CW}} \simeq 40 \mathrm{~nm}$ (a), and the dip $L_{\mathrm{CW}} \simeq 110 \mathrm{~nm}$ (b) net charge current of Fig. 8(a) in the case of $B=0.2 \mathrm{~T}$. Other parameters are $a_{w}=32.78 \mathrm{~nm}$, and $R_{\text {Dot }} \approx 25 \mathrm{~nm}$.

In Fig. 11 (b), the electrons are mostly localized in the control-QD corresponding to the current-dip at $L_{\mathrm{CW}} \simeq$ $110 \mathrm{~nm}$. This electron localization is expected because at a larger CW length the splitting of energy levels increases and leads to suppression the inter-waveguide scattering. Therefore, the electrons move along the controlwaveguide and get localized in the control-QD at this higher magnetic field.

\section{CONCLUDING REMARKS}

We have presented a model for a window coupled double quantum waveguide in an external perpendicular magnetic field. The DQW is weakly connected to two leads with different chemical potentials in which a non-Markovian master equation is utilized to investigate coherent switching in electron transport between the waveguides for the implementation of quantum logic gates.

A comparison between the charge current and the left and right currents in Figures 6 and 8 at the time point in time $t=200$ ps shows that the system has not completely reached a steady state yet, but the sought after function of the DQW is already present in the late transition regime. This is important. The exact steady state takes a long time to reach due to a low rate of charging for 2 and 3 electron states. Valuable time can be gained by operation of a device in the transition regime.

By tuning the length of the $\mathrm{CW}$, we have demonstrated two important physical characteristics of the waveguide system: resonant energy-levels and oscillations in the net charge current. The resonant energy levels indicate a strong coupling between the DQW and the oscillations denote that the resonance conditions are governed by the length of the CW.

In the case of an ideal non-Coulomb-interacting window-coupled DQW system, the charge current density splits equally between the waveguides at resonant energies, therefore, the net charge current reaches it's maximum value and the waveguide system works as a $\sqrt{\mathrm{NOT}}$-operation qubit. In the presence of strong magnetic field, the inter-waveguide backward scattering is enhanced, and the efficiency of the logic gate decreases.

This inter-waveguide forward scattering at resonant levels without a magnetic field is not seen without including two-electrons states in the model. It is strongly reduced by the Coulomb interaction that lifts the $2 \mathrm{ES}$ out of the group of active states in the transport. This aspect can though be controlled by the exact system size and design as the Coulomb blocking is reduced in a larger system, or by using a higher bias window or different material. Our models only includes sequential tunneling of electrons from the leads to the system, but the addition of higher order tunneling would only increase the importance of the many-electron structure and the Coulomb interaction on the operation of the system.

Two parallel waveguides or dots can be coupled in many distinct ways. Moldoveanu[16] et al. attain it capacitively, while Zibold[22] et al. fine tune the system by tunneling coupling. Both groups stress the strong dependence of the device on its exact geometry and the structure and correlations of many-electron states that are active in the transport. Our investigation with a different coupling scheme for the waveguides confirms this sensitive dependence.

In the presence of side QDs, more energy-levels of the two waveguides are brought into resonance which can 
lead to stronger coupling between them. Effectively, the dots can increase the density of states around the resonant transport states. The electrons from the controlwaveguide switch to the target-waveguide indicating a Not-operation qubit. But on the other hand, the sidedots can also increase the inter-waveguide backward scattering and reduce the qubit efficiency. In a stronger external magnetic field, the electrons get localized in the dots as the effective magnetic length is comparable to the radius of the side dot. In this case, the geometry of the CW does not affect the electron transport anymore.

The external magnetic field here is considered static, but one more variable to influence the transport is the shape of the side QDs in the waveguides. We show that the varying of the $\mathrm{CW}$ length between the waveguides can specify the type of the qubit logic gate to be implemented by the system.

\section{ACKNOWLEDGMENTS}

This work was financially supported by the Icelandic Research and Instruments Funds, the Research Fund of the University of Iceland, the Nordic High Performance Computing facility in Iceland, and the National Science Council in Taiwan through Contracts No. NSC1002112-M-239-001-MY3 and No. MOST103-2112-M-239001-MY3.
[1] P. Zhang, Z.-L. Xiang, and F. Nori, Phys. Rev. B 89, 115417 (2014).

[2] R. Ionicioiu, G. Amaratunga, and F. Udrea, Int. J. of Mod. Phys. B 15, 125 (2001).

[3] J. M. Martinis, S. Nam, J. Aumentado, and C. Urbina, Phys. Rev. Lett. 89, 117901 (2002).

[4] P. Bordone, A. Bertoni, M. Rosini, and C. Jacoboni, Semicond.Sci.Technol. 19, 412 (2004).

[5] L. E. Reichl and M. G. Snyder, Phys. Rev. A 72, 032330 (2005).

[6] M. J. Gilbert, R. Akis, and D. K. Ferry, Appl. Phys. Lett. 81, 4284 (2002).

[7] J. Harris, R. Akis, and D. K. Ferry, Appl. Phys. Lett. 79, 2214 (2001).

[8] M. G. Snyder and L. E. Reichl, Phys. Rev. A 70, 052330 (2004).

[9] A. Marchi, A. Bertoni, S. Reggiani, and M. Rudan, IEEE Transaction on nanotechnology 3, 129 (2003).

[10] A. Bertoni, P. Bordone, R. Brunetti, C. Jacoboni, and S. Reggiani, Phys. Rev. Lett. 84, 5912 (2000).

[11] A. Ramamoorthy, R. Akis, and J. P. Bird, IEEE Transaction on nanotechnology 5, 712 (2006).

[12] V. Moldoveanu, A. Manolescu, and V. Gudmundsson, New Journal of Physics 11, 073019 (2009).

[13] V. Gudmundsson, C. Gainar, C.-S. Tang, V. Moldoveanu, and A. Manolecu, New J. Phys.
11, 113007 (2009).

[14] V. Moldoveanu, A. Manolescu, C.-S. Tang, and V. Gudmundsson, Phys. Rev. B 81, 155442 (2010).

[15] N. R. Abdullah, C.-S. Tang, and V. Gudmundsson, Phys. Rev. B 82, 195325 (2010).

[16] V. Moldoveanu, A. Manolescu, and V. Gudmundsson, Phys. Rev. B 82, 085311 (2010).

[17] H.-P. Breuer and F. Petruccione, The Theory of Open Quantum Systems (Oxford University Press, Oxford, 2002).

[18] F. Haake, Phys. Rev. A 3, 1723 (1971).

[19] F. Haake, Quantum Statistics in Optics and Solid-state Physics, edited by G. Hohler and E.A. Niekisch, Springer Tracts in Modern Physics Vol. 66 (Springer, Berlin, Heidelberg, New York, 1973, p. 98.).

[20] N. R. Abdullah, C. S. Tang, A. Manolescu, and V. Gudmundsson, Journal of Physics:Condensed Matter 25, 465302 (2013).

[21] S. Datta, Electronic Transport in Mesoscopic system (Cambridge University Press, Cambridge, 1995).

[22] T. Zibold, P. Vogl, and A. Bertoni, Phys. Rev. B 76, 195301 (2007).

[23] J. Gong, F.-H. Yang, and S.-L. Feng, Chin. Phys. Lett. 24, 2383 (2007).

[24] G. B. Akguc, L. E. Reichl, A. Shaji, and M. G. Snyder, Phys. Rev. A 69, 042303 (2004). 